\title{
Cyclic Hematopoiesis: the Mechanism of Cyclic Neutropenia in Grey Collie Dogs
}

\author{
David C. Dale, David W. Alling, and Sheldon M. Wolff \\ From the Department of Health, Education and Welfare, National Institutes \\ of Health, National Institute of Allergy and Infectious Diseases, Laboratory \\ of Clinical Investigation, and Office of the Scientific Director, \\ Bethesda, Maryland 20014
}

A B S T R A C T Two grey collie dogs had regular cyclic fluctuations in the number of all formed elements of the blood. The period lengths for all elements for an individual dog were the same, but the pattern of fluctuation for each element was distinctive. Normal dogs lacked periodic fluctuations.

The patterns of day-to-day variation in the normal dogs counts were consistent with a first-order autoregressive process of serial dependence (i.e., each observation of the series depends on the last preceding observation and no others). The grey collie counts showed the same pattern of serial dependence after the component of the over-all variability due to cyclic oscillation was removed. These data suggest that a defect of hematopoietic regulation at the stem cell level leads to periodic interruptions of production of all hematopoietic elements and accounts for the cycles seen in the peripheral blood counts.

\section{INTRODUCTION}

Collie dogs with a distinctive grey coat color have cyclic neutropenia. At about 12-day intervals, they become severely neutropenic and frequently have fever, anorexia, and serious, often fatal, infections. Because the clinical and hematologic features of grey collie and human cyclic neutropenia are so similar, these dogs provide a suitable model for investigation of this disorder $(1,2)$.

The etiology of cyclic neutropenia is not known. One possible mechanism is that it occurs as an exaggeration of the normal fluctuation of blood neutrophil (PMN) ${ }^{1}$ counts (3). According to this hypothesis, the number of circulating PMN is stabilized by two negative feedback

\footnotetext{
Received for publication 29 November 1971 and in revised form 9 March 1972.

${ }^{1}$ Abbreviations used in this paper: PMN, blood neutrophils; RBC, red blood cells; WBC, white blood cells.
}

control loops, one regulating production and the other regulating the release of mature PMN from the bone marrow reserve. The feedback loop regulating production has a response time of 7-12 days; hence, if blood PMN were regulated by this loop alone, large oscillations in blood PMN counts would occur. Normally, PMN can be rapidly released from the large marrow PMN reserve and the controlled release of these cells tends to damp the natural oscillations in PMN production. Cyclic neutropenia results if the damping mechanism fails or the marrow PMN reserve is moderately decreased by a mild failure of PMN production (4-6). Other mechanisms of cyclic neutropenia have been suggested, such as an effect of the cyclic hormonal fluctuations in the menstrual cycle (7), a steroid hormone imbalance (8), splenic dysfunction $(8,9)$, and a process leading to intermittent maturational failure (10). Recent studies of PMN production and the half-life of blood PMN $(11,12)$ as well as serial marrow morphologic examination (9-11) indicate that cyclic neutropenia is more likely due to periodic interruptions of PMN production.

In the course of our studies of cyclic neutropenia in grey collie dogs, we have observed that not only the number of blood PMN but also the number of circulating monocytes, lymphocytes, eosinophils, reticulocytes, and platelets fluctuate cyclically. The pattern of fluctuation for each element is distinctive, although the cycle length for each is essentially the same. These observations suggest that the periodic failure of PMN production is part of a basic hematopoietic regulatory defect which probably affects cell production at the pluripotential stem-cell level.

\section{METHODS}

Dogs. Two grey collies (one male, aged 3 months and one female, aged $1 \mathrm{yr}$ ) and two normal collies (one male, aged 3 months and one female, aged 4 months) with no 
TABLE I

Periodogram Calculations

\begin{tabular}{|c|c|c|c|c|c|c|}
\hline Dog & Neutrophil & Eosinophil & Monocyte & Lymphocyte & Platelet & Reticulocyte \\
\hline \multicolumn{7}{|l|}{ Grey collie 2} \\
\hline Study length, days & 162 & 162 & 162 & 162 & 78 & 29 \\
\hline Estimated period, days* & 13.1 & 13.5 & 13.5 & 13.5 & 13.0 & 14.5 \\
\hline$g \ddagger$ & 0.236 & 0.129 & 0.196 & 0.093 & 0.412 & 0.151 \\
\hline$P \S$ & $<0.001$ & $<0.01$ & $<0.001$ & 0.04 & $<0.01$ & $>0.05$ \\
\hline \multicolumn{7}{|l|}{ Grey collie 12} \\
\hline Study length & 162 & 162 & 162 & 162 & 78 & 59 \\
\hline Estimated period & 11.2 & 10.8 & 11.6 & 11.6 & 11.1 & 11.8 \\
\hline g & 0.214 & 0.112 & 0.186 & 0.087 & 0.571 & 0.560 \\
\hline$P$ & $<0.001$ & $<0.01$ & $<0.001$ & 0.06 & $<0.01$ & $<0.01$ \\
\hline \multicolumn{7}{|l|}{ Normal collie 15} \\
\hline Study length & 118 & 118 & 118 & 118 & $\mathrm{ND} \|$ & 96 \\
\hline Estimated period & 9.8 & 14.8 & 2.4 & 11.8 & $\mathrm{ND}$ & 32.0 \\
\hline $\mathrm{g}$ & 0.09 & 0.093 & 0.098 & 0.102 & ND & 0.200 \\
\hline$P$ & 0.20 & 0.23 & 0.16 & 0.13 & ND & $<0.01$ \\
\hline \multicolumn{7}{|l|}{ Normal collie 16} \\
\hline Study length & 124 & 124 & 124 & 124 & ND & 46 \\
\hline Estimated period & 14.6 & 31.0 & 5.4 & 31.0 & ND & 13.7 \\
\hline $\mathrm{g}$ & 0.096 & 0.123 & 0.056 & 0.093 & ND & 0.158 \\
\hline$P$ & 0.15 & 0.02 & $>0.50$ & 0.17 & ND & $<0.01$ \\
\hline
\end{tabular}

* Estimated period is the period corresponding to the largest periodogram peak.

$\ddagger g$ is the Fisher statistic, see text.

$\S P$ is the level of significance.

II ND is not done.

history of grey forebears were housed in air-conditioned quarters and similarly studied. $1-\mathrm{ml}$ venous blood samples were drawn daily at 1:00 p.m. from the cephalic veins.

Blood counts. White blood cell (WBC), red blood cell (RBC), and platelet counts were made on EDTA-anticoagulated samples using an electronic particle counter ${ }^{2}$ and standard techniques (13-15). 100 cell differential counts were done on air-dried, Wright's-stained smears. For reticulocyte counts, two drops of EDTA-anticoagulated blood and two drops of $N$-methylene blue stain (Brecher formula) ${ }^{3}$ were mixed for $10 \mathrm{~min}$ and air-dried smears made. The percentage of reticulocytes was determined by examining $1000 \mathrm{RBC}$ in adjacent microscopic fields using a lined microscopic field or a Miller disc ${ }^{4}$ to facilitate counting. All of the smears were examined by either of two investigators whose agreement on differential counts and reproducibility of individual counts were within the expected statistical limits $(16,17)$.

Statistical methods. The serial blood counts of the normal and grey collies were analyzed as follows: the sequence of counts was assumed to be a series of chance deviations from a process of strictly regular oscillations. The oscillations were assumed to be a combination of sine and cosine curves (a trigonometric regression function)

${ }^{2}$ Coulter counter model Fn, Coulter Electronics, Industrial Div., Hialeah, Fla.

${ }^{3}$ Hartman-Leddon Company, Philadelphia, Pa.

* Bausch \& Lomb, Inc., Rochester, N. Y. and the deviations from these oscillations, to be a stationary time series, that is, a series of values equidistantly spaced in time among which there were no trends or other evolutionary changes.

First, in order to obtain a series of values equally spaced in time, the occasional gaps which occurred in the sets of observations (3-9\% of the total observations for the various series) were filled in by linear interpolation. Next, the cell counts of zero were changed to ones and then all counts were converted to logarithms so that cell count distributions would tend to be symmetrical around the mean and, hence, could be considered approximately normally distributed (zero counts constituted less than $2 \%$ of all PMN and monocyte counts and $8-30 \%$ of all reticulocyte and eosinophil counts). The logged data were then centered at zero. Finally, because trends and fluctuations with periods of greater than about 60 days would seriously hamper the detection of periods of 50 days or less, the data were then smoothed with a fourth-degree polynomial to remove such trends.

After this preliminary centering and smoothing, the Schuster periodogram (18) was calculated in order to detect periodicity in the data. Roughly speaking, the ordinate of the periodogram shows the amount of variability that oscillations of each period length contribute to the total variability. The significance of the largest peak of the periodogram was determined by the Fisher statistic $(\mathrm{g})$, which is the ratio of the height of this peak to the area between the periodogram and the horizontal axis (19). In those 
animals where the periodogram showed significant periodicity in each of the cell types, the corresponding period lengths were averaged and the integer nearest to the average was taken to be the period common to all cell types. The coefficients of the trigonometric regression function for each cell type were then estimated by Fourier analysis (20). The regression functions were then subtracted from the smoothed data leaving what was assumed to be a stationary time series.

The hypothesis that the time series in question is a firstorder autoregression, that is, that each daily count depends on the previous day's count, plus some independent random variable, was then tested using Quenouille's procedure (21). In the latter test, autoregressive processes up to order 21 were considered as alternatives (i.e., the degrees of freedom equal 20). A first-order autoregression can be expressed as $x_{\mathrm{t}}=\rho x_{\mathrm{t}-1}+e_{\mathrm{t}},-1 \leqq \rho \leqq 1$, where $x_{\mathrm{t}}$ is the $\mathrm{t}^{\text {th }}$ smoothed log count from which the periodic component has been removed; $e_{t}$ is an independent normal random variable and $\rho$ is the correlation between $x_{t}$ and $x_{\mathrm{t}-1}$.

\section{RESULTS}

Serial leukocyte, platelet, and reticulocyte counts were made in each of two grey collies for the intervals of time shown in Table I. For the two normal collies, daily
WBC and differential counts and reticulocyte counts were made for intervals ranging from 96 to 124 consecutive days (Table I).

Fig. 1 shows the daily counts of PMN, eosinophils, monocytes, lymphocytes, platelets, and reticulocytes for grey collie 12 during the entire period of study. There were similar findings in grey collie 2 and also in the other grey collies previously studied. Certain features of the individual curves and their interrelationships are noteworthy. For the PMN, after the recovery from neutropenia, there is usually a transient period of neutrophilia, then a dip, and second peak of PMN's before neutropenia recurs (22). Eosinophils and monocytes are increased during the neutropenic period. In this dog, the eosinophil fluctuations are not so striking as in some other grey collies, but the timing of the increase in eosinophils has been the same in all of these dogs. At the time of the peak of blood monocytes, myelocytes, metamyelocytes, and band PMN are also seen in the peripheral blood. The monocytes generally are morphologically distinguishable from the PMN precursors by light microscopy, and the presence of both types of cells



Figure 1 Blood counts of grey collie 12. Absolute WBC, platelet, and reticulocyte counts in a grey collie dog are plotted for the 162 day period of study and show cyclical fluctuation with a period of 11 days. 

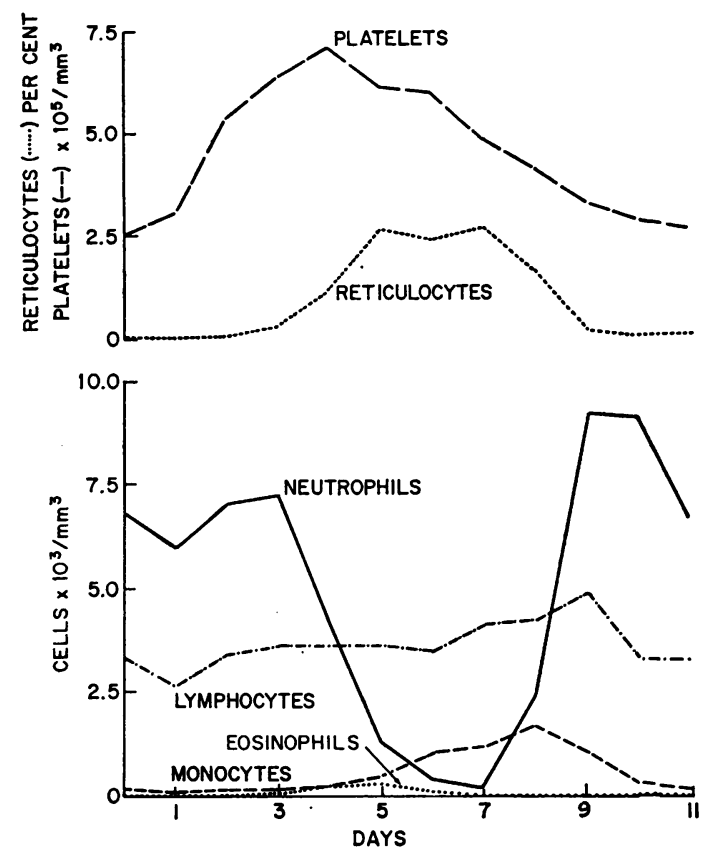

Figure 2 Mean cell counts for the 11 day cycle. Geometric mean cell counts were calculated from 14 successive cycles in a single grey collie dog (12) and plotted.

has been confirmed by electron microscopy. ${ }^{5}$ The reticulocyte count is also regularly increased in the grey collies during the neutropenic phase. Between the reticulocyte peaks, the reticulocyte count falls to nearly zero. Cyclic fluctuations in the RBC count and hematocrit (not shown) also occur with the increase in these values occurring immediately subsequent to the reticulocytosis. The platelet count also shows striking cyclic variation. The nadir of the platelet count occurs when the neutrophil count is normal, but soon the platelets begin to rise, the rise continuing until early in the neutropenic period; thereafter, platelets fall for a 3 or 4 day period during which the PMN are increasing.

Fig. 2 shows the geometric mean counts for each of the cell types in one grey collie for corresponding days of 14 successive 11-day cycles. It can be seen that each element has its distinctive cyclic pattern. The day on which the lowest average platelet count occurred is taken as day zero. With this reference point, the peak counts for platelets, reticulocytes and eosinophils, monocytes and PMN successively occur.

The blood cell counts of the normal collies show no such regular variation as is seen in the series of counts from the grey collie illustrated in Figs. 1 and 2.

To compare more precisely the periodicity in the grey and normal collie blood counts, the Schuster periodogram

\footnotetext{
${ }^{5}$ Scott, R. E., D. C. Dale, and A. Rosenthal. In preparation.
}

was calculated for each blood element for the four dogs; the periodograms for one grey and one normal are shown in Fig. 3. For all blood elements, the grey collie shows sharp peaks corresponding to an average period length of 11.35 days. There are also peaks for the grey collie counts corresponding to periods of 5.5 days particularly noticeable for PMN and eosinophils and 44 days for eosinophils and platelets. The normal collie shows no such large peak for any cell type. Table I shows the estimated dominant period length for the grey and normal collies for each element. For the grey collies, the largest peak values were significant $(P<0.05)$ for all cell types except reticulocytes in one dog where the period of observation was very short.

For the normal collies, significant peaks could be identified for reticulocytes in one dog and for eosinophils and reticulocytes in the other. These periodicities can be regarded, however, as spurious as discussed below. For the normal dogs the largest peaks for the various elements also showed very little tendency to coincide, which is in contrast to the almost complete coincidence found in the grey collies. Fourier analysis, based on the common period length for the regular oscillations in the grey collie blood counts, was used to estimate the coefficients of the trigonometric regression functions which were then subtracted from the log counts in order to remove the effect of the regular oscillatory process from the over-all variability. Not all of the day-to-day variation was explained by the oscillatory process as is illustrated for PMN of one dog in Fig. 4. The area between the fitted trigonometric function curve and the observed values represents the variability not explained by the regular oscillatory process.

Since we failed to observe consistent cyclic variation in most of the blood elements of the normal dogs and since a substantial portion of the variation in the grey collie counts could not be attributed to the oscillatory process, the data were examined to determine whether an autoregressive process could explain the day-to-day variation of the blood cell counts. The simplest process of this type is a first-order autoregression; that is, a series of observations, each of which depends on the last preceding observation and no others. The strength of the dependence in question is measured by the serial correlation coefficient $(\rho)$; accordingly, this parameter was estimated from each set of smoothed data for the normal collies and from the smoothed data with the oscillatory component removed for the grey collies. In Table II are shown the values of the correlation coefficient, $\rho$, for each of the cell types for the four dogs. It is seen that, in the case of the neutrophils, lymphocytes, and eosinophils, there is only moderate serial correlation without any apparent systematic difference between grey collies and normal dogs. In the case of the monocytes 

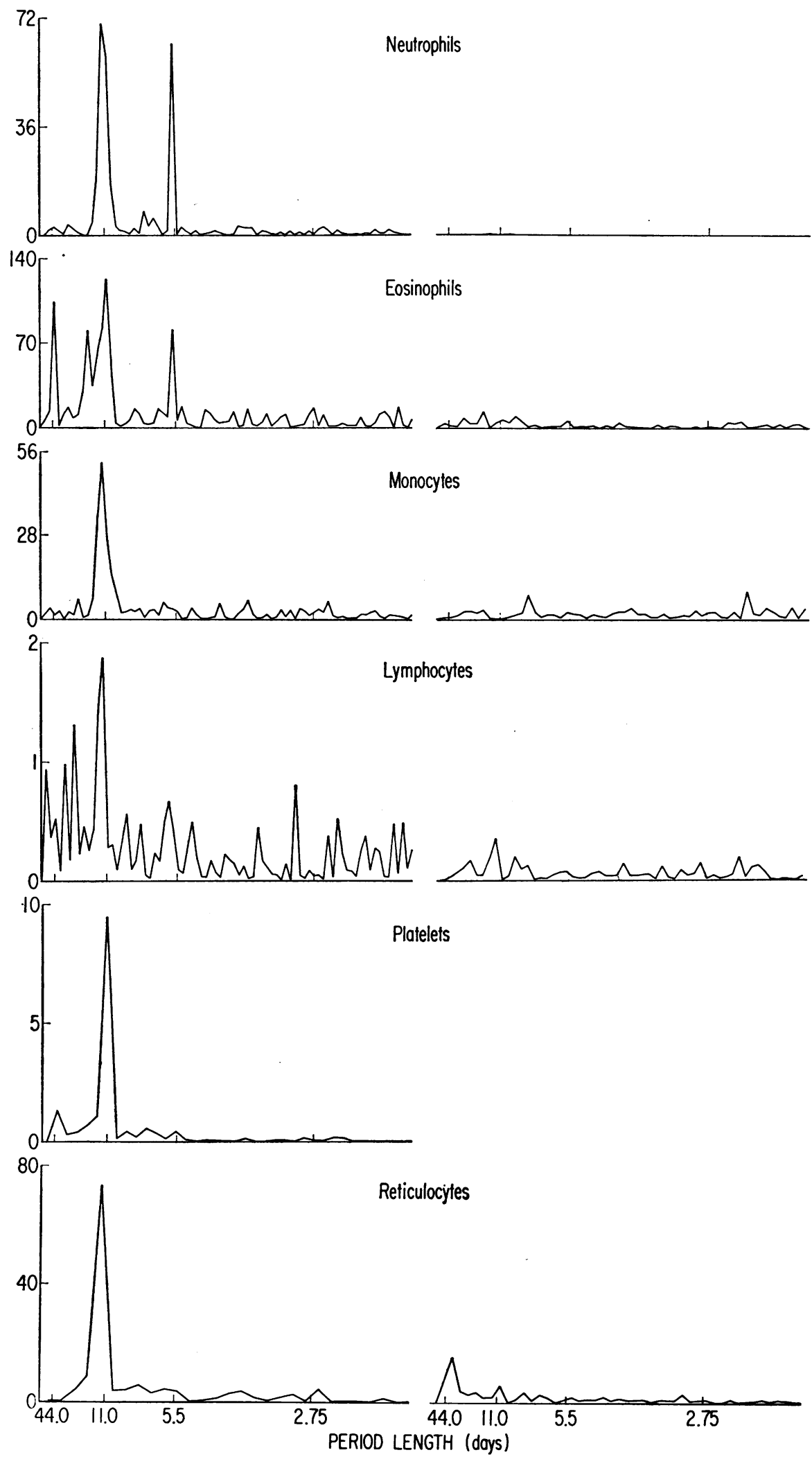

FIgURE 3 Periodograms of serial blood counts. Comparison of periodograms for various blood cells of grey collie 12 (left) and normal collie 15 (right). Note 11 day cycle for all blood elements in the grey collie with harmonics in some elements at 5.5 and 2.75 days. 


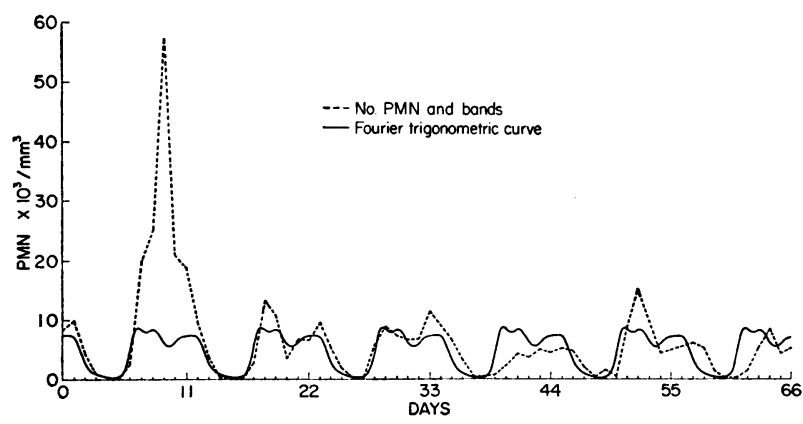

Figure 4 Trigonometric curve (solid line) fitted to serial daily polymorphonuclear leukocyte and band counts (dashed line).

and reticulocytes, there may be differences between the grey and normal collies; however, this impression needs to be confirmed by the acquisition of more data.

The autoregression model appears to be an adequate representation of the data in view of the fact that goodness of fit (Quenouille) tests failed to show significant lack of fit except for the PMN of normal collie 16 ( $P=$ 0.02 ). (The latter circumstance is not unexpected in view of the number of tests of significance that have been computed.) This is the type of process one would expect with a linear negative feedback system which tightly controlled the counts of the various elements near their mean values.

\section{DISCUSSION}

These studies indicate that in grey collie dogs there are regular cyclic fluctuations in the number of blood PMN, monocytes, lymphocytes, eosinophils, platelets, and reticulocytes and that the cycles are the same length for all elements for an individual dog. The two normal collie dogs show remarkably less variability in PMN, monocytes, and eosinophils; moreover, there is little suggestion in either normal dog of concurrent cyclic variation in any of the five cell types studied. The striking differences in the magnitude and patterns of blood cell fluctuation between grey and normal collies clearly distin- guishes the grey collie hematopoietic system as distinctly abnormal.

These data provide some new information about the mechanism of cyclic neutropenia and the regulation of hematopoiesis. It is clear that the levels of the various blood elements of the grey collies do not rise and fall concomitantly but rather follow entirely separate patterns. If the numbers of the blood elements did fall concomitantly, this would suggest a peripheral mechanism of cell removal or sequestration. If the cyclic variation in the number of each cell were due to some intrinsic rhythm of production characteristic for each cell type, then from what is known of the differences in the production times of PMN, RBC, and platelets (23-25), it would seem quite unlikely that the cyclic fluctuations of the various cells would remain synchronous over long periods of time.

The possibility that cyclic neutropenia is a result of the failure of the damping effect of the marrow reserve (4-6) also seems unlikely. First, these data indicate that normal collie PMN counts follow a pattern consistent with a first-order autoregression as would be expected if a linear negative feedback control of the blood PMN count operated through the rapid release of cells from the marrow reserve. The PMN of the grey collies have the same pattern of variation if the effect of the regular oscillatory process, which is probably due to the periodic fluctuation in PMN production (22), is removed. This strongly suggests that their marrow reserve mechanism is intact. Previously, direct measurements of the bone marrow PMN reserves of grey collies have been made (22). These studies indicated that, when grey collies have normal blood PMN counts, marrow reserves are readily measurable and that neutropenia occurred only after the PMN reserves were exhausted. Furthermore, this kind of a mechanism does not explain the cyclic variation in the other elements for which there is no known marrow reserve.

Although it is conceivable that the cyclic variation in the cell counts might represent a nonspecific interrelationship, for example, repeated neutropenia leading to infection and secondarily repeated suppression of erythropoiesis; the consistency over many cycles in the tim-

TABLE II

Correlation Coefficients ( $\rho$ ) of the First-Order Autoregressions

\begin{tabular}{lcccccc}
\hline \multicolumn{1}{c}{ Dog } & Neutrophil & Eosinophil & Monocyte & Lymphocyte & Platelet & Reticulocyte \\
\hline Grey collie 2 & 0.290 & 0.248 & 0.594 & 0.262 & 0.509 & 0.016 \\
Grey collie 12 & 0.321 & 0.427 & 0.274 & 0.262 & 0.574 & 0.219 \\
Normal collie 15 & 0.212 & 0.173 & -0.078 & 0.160 & ND $^{*}$ & 0.541 \\
Normal collie 16 & $0.318 \ddagger$ & 0.285 & 0.098 & 0.301 & ND & 0.485 \\
\hline
\end{tabular}

* Not done.

$\ddagger$ There is a significant lack of fit between this series of counts and a first-order autoregression. 
ing and patterns of the interrelationship, for example, reticulocytosis in the neutropenic phase or thrombocytosis during the normal phase, do not support this possibility.

The most reasonable explanation for the periodic fluctuations in the various hematopoietic elements of the grey collie is that production of all blood elements fails periodically at an early stage in the development of these cells. Thus, if all hematopoietic cell production were transiently "turned off" at the stem-cell level at about the time of the peak of neutrophilia and if marrow proliferation of these cells were "turned on" a day or so later; then the sequence of a rising platelet count, reticulocyte count, monocyte count, and the PMN count would be seen. Evidence favoring this intermittent failure of production has been previously presented for PMN (22). Periodic fluctuations in the relative number of marrow RBC precursors (22) and in the tritiated thymidine labeling of erythroid precursors has also been observed (26). The marrow histologic examinations indicate that the erythroid and myeloid proliferations occur concomitantly and suggest that the reticulocytosis precedes the neutrophilia due to the difference in the maturation times for these elements. The present inadequacy of our understanding of the regulation of hematopoiesis and particularly the factors governing stem-cell proliferation, however, make this hypothesis only tentative. Certainly other possibilities, such as a competition for a limited number of stem cells to differentiate along the myeloid, erythroid, or megakaryocytic lines (27) and the possibility that the marrow environment is in some ways inadequate to maintain steady cell production, should be considered (28). Nevertheless, these observations suggest that the defect in these dogs either results from an abnormality of their pluripotential stem cells' capacity to be triggered into commitment and differentiation or is a consequence of some factor regularly interfering with the proliferation of the hematopoietic precursor cells. An inhibitory hormone for hematopoiesis or "chalone" has previously been proposed and measured $(29,30)$, and it is intriguing to speculate that an intermittent excess of this factor is the cause for the cyclic marrow failure.

The serial blood cell counts of the two normal collies with unperturbed hematopoiesis showed little evidence that hematopoiesis is normally periodic. The fact that, except for neutrophils in one dog, the serial counts of the normal dogs are consistent with a first-order autoregressive process indicates that the periodicities suggested by the Schuster periodogram for the normal dogs are spurious since an essential property of the first-order autoregression is the lack of periodicity (31). This deficiency of the periodogram, that is, its capacity to indicate spurious periodicities has not been generally recognized in studies of this kind. These data on normal dogs thus indicate that canine blood elements are closely regulated.

Studies of cyclic neutropenia in man have generally reported cyclic variation only in PMN and monocytes $(10,11)$. Although a few patients have also had cyclic fluctuation in platelet counts (32), insufficient information is presently available to decide if cyclic neutropenia in man is due to as generalized a defect as suggested for grey collies dogs.

\section{ACKNOWLEDGMENTS}

We thank Mr. Stanley Ward and Miss Esther Graham for their technical assistance and Mr. R. J. Romanoff of the Hybrid Computer Group, DCRT, for valuable help in programming and performing computation on the CDC 3100 computer.

\section{REFERENCES}

1. Lund, J. E., G. A. Padgett, and R. L. Oh. 1967. Cyclic neutropenia in grey collie dogs. Blood J. Hematol. 29: 452.

2. Dale, D. C., H. R. Kimball, and S. M. Wolff. 1970. Studies of cyclic neutropenia in grey collie dogs. Clin. Res. 18 : 402. (Abstr.)

3. Morley, A. A. 1966. A neutrophil cycle in healthy individuals. Lancet. 2: 1220.

4. Morley, A. A., E. A. King-Smith, and F. Stohlman, Jr. 1970. The oscillatory nature of hematopoiesis. In Hematopoietic Cellular Proliferation. F. Stohlman, Jr., editor. Grune \& Stratton, Inc., New York. 3.

5. Morley, A. A. 1970. Periodic diseases, physiologic rhythms and feedback control-a hypothesis. Australas. Ann. Med. 3 : 244.

6. Morley, A. A., and F. Stohlman, Jr. 1970. Cyclophosphamide-induced cyclic neutropenia: an animal model of a human periodic disease. N. Engl. J. Med. 282: 643.

7. Thompson, W. P. 1934. Observations on the possible relationship between agranulocytosis and menstruation with further studies on a case of cyclic neutropenia. $N$. Engl. J. Med. 210: 176.

8. Reznikoff, P. 1946. Cyclic neutropenia: a case study with bone marrow findings before and after splenectomy. Trans. Assoc. Am. Physicians Phila. 59: 276.

9. Fullerton, H. W., and H. L. D. Duguid. 1949. A case of cyclic agranulocytosis with marked improvement following splenectomy. Blood J. Hematol. 4: 269.

10. Page, A. R., and R. A. Good. 1957. Studies on cyclic neutropenia. Am. J. Dis. Child. 94: 623.

11. Meuret, G., and T. M. Fliedner. 1970. Zellkinetik der Granulopoese und des Neutrophilensystems bei einem Fall von zyklisher Neutropenie. Acta Haematol. (Basel). $43: 48$.

12. Dienard, A. S., and A. R. Page. 1970. An improved method for performing neutrophil survival studies. Blood J. Hematol. 36 : 98.

13. Richar, W. J., and E. S. Breakell. 1959. Evaluation of electronic particle counter for counting white blood cells. Am. J. Clin. Pathol. $31: 384$.

14. Brecher, G., M. A. Schneiderman, and G. Z. Williams. 1956. Evaluation of electronic red blood cell counter. Am. J. Clin. Pathol. 26: 1439.

Cyclic Hematopoiesis in Grey Collie Dogs 2203 
15. Bull, B. S., M. A. Schneiderman, and G. Brecher. 1965. Platelet counts with the Coulter counter. Am. J. Clin. Pathol. 44 : 678.

16. Barnett, C. W. 1933. Unavoidable errors in differential count of leukocytes of blood. J. Clin. Invest. 12: 77.

17. Garwood, F. 1936. Fiducial limits for the Poisson distribution. Biometrika. $28: 437$.

18. Hannan, E. J. 1960. Estimation of the spectral density and distribution functions. Time Series Analysis. Methuen \& Co. Ltd., London. 52.

19. Fisher, R. A. 1929. Tests of significance in harmonic analysis. Proc. Roy. Soc. London. 125: 54.

20. Aitken, A. C. 1957. The method of least squares: multivariate correlation: polynomial and harmonic regression. Statistical Mathematics. Oliver \& Boyd Ltd. Edinburgh. 106.

21. Quenouille, M. H. 1947. A large-sample test for the goodness of fit of autoregressive schemes. J. Roy. Stat. Soc. 110: 123.

22. Dale, D. C.. S. B. Ward, H. R. Kimball, and S. M. Wolff. 1972. Studies of neutrophil production and turnover in grey collie dogs with cyclic neutropenia. J. Clin. Invest. 50: 0000 .

23. Wintrobe, M. M. 1966. Clinical Hematology. Lea \& Febiger, Philadelphia. 20-21.

24. Cronkite, E. P., and T. M. Fliedner. 1964. Granulocytopoiesis. N. Engl. J. Med. 270: 1347.
25. Odell, T. T., Jr., and C. W. Jackson. 1970. Megakaryocytopoiesis. In Hemopoietic Cellular Proliferation. F. Stohlman, Jr., editor. Grune \& Stratton, Inc., New York. 278.

26. Lund, J. E., and M. A. Maloney. 1969. Erythrocyte production in canine cyclic neutropenia. Fed. Proc. 28 : 685. (Abstr.)

27. Hellman, S., and H. E. Grate. 1967. Hematopoietic stem cells: evidence for competing proliferative demands. Nature (Lond.). 216: 65 .

28. McCulloch, E. A., L. Siminovitch, J. E. Till, E. S. Russell, and S. E. Bernstein. 1965. The cellular basis of the genetically determined hematopoietic defect in anemic mice of genotype S1/S1 d. Blood J. Hematol. 26: 399.

29. Rytömaa, T., and K. Kiviniemi. 1968. Control of granulocyte production. I. Chalone and antichalone, two specific humoral regulators. Cell Tissue Kinet. 1: 329.

30. Paran, M., Y. Ichikawa, and L. Sachs. 1968. Feedback inhibition of the development of macrophage and granulocyte colonies. II. Inhibition by granulocytes. Proc. Nat. Acad. Sci. U. S. A. $62: 81$.

31. Bartlett, M. S. 1955. An Introduction to Stochastic Processes. Cambridge University Press, New York. 257.

32. Liu, Y. K., W. Adams, and L. W. Sullivan. 1970. Periodic neutropenia associated with periodic fluctuations in platelet counts. Clin. Res. 18: 409. (Abstr.) 